CLINICAL STUDY

\title{
Diagnostic accuracy of free and total metanephrines in plasma and fractionated metanephrines in urine of patients with pheochromocytoma
}

\author{
Eric Grouzmann, Laurence Drouard-Troalen ${ }^{1,2}$, Eric Baudin ${ }^{1,2}$, Pierre-François Plouin ${ }^{3}$, Beat Muller ${ }^{4}$, \\ Daniela Grand and Thierry Buclin \\ Division of Clinical Pharmacology and Toxicology, University Hospital, 1011 Lausanne, Switzerland, Departments of ${ }^{1}$ Clinical Biology and ${ }^{2}$ Radiology, \\ Institut Gustave-Roussy, Villejuif, France, ${ }^{3}$ Hypertension Unit, AP-HP, Hôpital Européen Georges Pompidou, Faculté de Médecine, Université Paris \\ Descartes, Paris, France and ${ }^{4}$ Division of Endocrinology, Diabetology and Clinical Nutrition, University Hospital, Basel, Switzerland \\ (Correspondence should be addressed to E Grouzmann; Email: eric.grouzmann@chuv.ch)
}

\begin{abstract}
Background: Plasma free and urinary metanephrines are recognized biomarkers for the assessment of pheochromocytoma. Plasma total metanephrines with a long half-life may represent another useful biomarker.

Objective: The aim of this study is to evaluate the diagnostic performances of plasma total metanephrines alone or combined with free metanephrines and fractionated 24-h urinary metanephrines.

Methods: A retrospective, case-control diagnostic test study was conducted between 1999 and 2007 in two university hospitals in Switzerland and two institutions in France. The patients included 46 cases with histologically proven pheochromocytoma, and 181 controls suspected of tumor with negative investigations and 3-year follow-up. None had renal dysfunction. Sensitivity and specificity were compared after expressing each measurement result as a ratio over its upper reference limit, adding the ratios of normetanephrine and metanephrine, and defining cut-off values of 1 or 2 for this sum.

Results: Applying a cut-off value of 1, plasma free and total metanephrines and urinary fractionated metanephrines had similar sensitivities of 96\% (95\% confidence interval, 86-99\%), 95\% (85-99\%), and 95\% (84-99\%) along with similar specificities of 89\% (83-94\%), 91\% (84-95\%), and 86\% $(80-91 \%)$. A cut-off of 2 for the sum of ratios over reference limit improves the specificity, and it can be used for a confirmation test based on another biomarker taken among the three biomarkers.

Conclusion: All three metanephrine-based tests perform equivalently for diagnosing pheochromocytoma in the absence of renal insufficiency, and can be conveniently associated two by two for confirming/excluding tumor.
\end{abstract}

European Journal of Endocrinology 162 951-960

\section{Introduction}

Pheochromocytoma, a rare tumor arising in chromaffin cells, is not diagnosed by clinical examination alone, making physicians often request biochemical screening for tumoral catecholamine secretion (1). However, its incidence is $<1-8 / 1000000$ per year, requiring sensitive and specific tests to reliably exclude or confirm its presence. Plasma catecholamines are still requested to detect a pheochromocytoma. Unfortunately, their short half-life makes it difficult to distinguish pathological overproduction from transient burst of secretion from nerve endings during the stress of blood sampling (2). Therefore, urinary fractionated metanephrines and more recently plasma free metanephrines have been proposed for the biochemical screening of pheochromocytoma (3). Lenders (4) \& Raber (5) view plasma free metanephrine determination as the 'gold standard' for pheochromocytoma diagnosis. However, their conclusions were challenged by Sawka, who found a relatively large number of false-positive results with this biomarker, and suggested instead to combine measurements of 24-h urinary metanephrines and catecholamines (6). However, the blood samples in that study were drawn in the sitting position, causing false-positive results when compared with the supine position used in the other studies $(4,7)$. Boyle found urinary free metanephrines to be superior to urinary catecholamines and plasma catecholamines, but did not evaluate them against urinary hydrolyzed metanephrines or plasma free metanephrines (8).

We have postulated that total (notice that the term total metanephrine is used here to designate the sum of free plus conjugated metanephrine; in older 
literature, the term total metanephrines was used to designate the sum of metanephrine and normetanephrine, when both compounds were assayed together; i.e. sulfate-conjugated plus free) plasma metanephrines might have a diagnostic interest due to their prolonged blood persistence (9). Their abundance in plasma is 10-20-fold higher than that of the free forms, and their determination is analytically easier (9). However, conjugated metanephrines have clearance rates that are different from those of free metanephrines, and can therefore increase in renal impairment (10), as it has been observed when creatininemia exceeds $120-170 \mu \mathrm{mol} / \mathrm{l}(11,12)$. High sensitivity and specificity of plasma total metanephrines have been reported previously for the diagnosis of pheochromocytoma, but without comparison against other tests available $(11,12)$. A study compared plasma and urinary metanephrines using a colorimetric urine test, which did not allow distinguishing metanephrine from normetanephrine, thus resulting in lower sensitivity (13). However, larger increases were found with plasma free metanephrines than with plasma total metanephrines in patients with tumors.

The aim of this study was to compare the diagnostic performances of plasma total metanephrines measurement with those of plasma free catecholamine and metanephrines and 24-h urinary fractionated metanephrines measurement in patients having surgically proven pheochromocytoma compared with controls without evidence of tumor.

\section{Materials and methods}

\section{Study design and participants}

This retrospective study included patients in whom plasma free and total metanephrines, plasma catecholamines, and urinary fractionated metanephrines were investigated for a suspicion of pheochromocytoma at

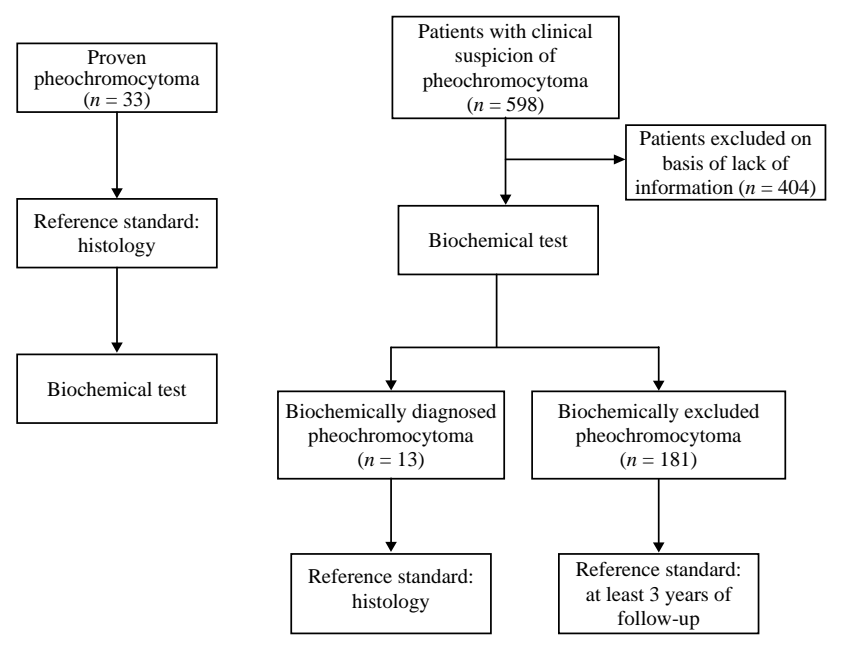

the Hôpital Européen Georges Pompidou (Paris, France), the Institut Gustave Roussy (Villejuif, France), the University Hospital of Lausanne (Switzerland), and the University Hospital of Basel (Switzerland; Fig. 1). All patients tested between 2003 and 2004 were considered for inclusion in the study, while supplemental pheochromocytoma patients were included between 1999 and 2007. Plasma free and total metanephrines, plasma catecholamines, and urinary fractionated metanephrines were all measured in Lausanne, while plasma catecholamines and urinary fractionated metanephrines were determined in the four centers. The hospital's institutional review board approved the study.

The biochemical determinations were also performed in 63 healthy subjects recruited as normal volunteers in pharmacological trials after full clinical and laboratory checkups. However, they were not included in the controls, as the study was conducted from an 'intention to diagnose' perspective, i.e. considering a target population in whom the biomarkers are to be measured in practice (14).

Controls consisted of three patient groups. The first group incorporated 133 patients with a clinical presentation suggestive of pheochromocytoma (hypertension difficult to treat with recent onset of headaches, tachycardia, sweating, or pallor paroxysms), for whom the disease was excluded by observing at least 3 years of negative clinical follow-up. They are typically representative of individuals from whom diagnostic tests are requested for suspicion of pheochromocytoma. The most common final diagnosis was essential hypertension; it also included menopause, idiopathic hyperhidrosis, Hashimoto's disease, renal carcinoma, renal artery stenosis, primary hyperaldosteronism, Cushing's disease, pituitary adenoma, Crohn's disease, Basedow/ Graves' disease, and panic attacks. The second group included 23 patients routinely screened for pheochromocytoma after the discovery of an adrenal incidentaloma (tumor-like adrenal image on abdominal

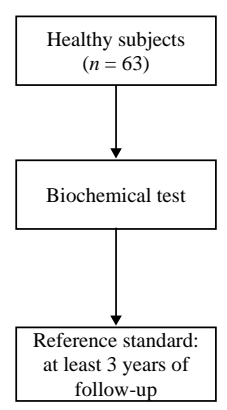

Figure 1 Flowchart of the patients included in the study (the 404 patients excluded from this study were referred from regional laboratories without information available). 
computed tomography scan or ultrasound requested for unrelated reasons). The last group consisted of 25 patients undergoing biochemical surveillance because of family history of pheochromocytoma (12 MEN2A, 11 MEN2B, 1 von Hippel-Lindau's (VHL) disease confirmed by germline identification of RET, SDH, and VHL mutations, and 1 neurofibromatosis established phenotypically).

Among the 46 patients with histologically proven pheochromocytoma, 29 had sporadic tumors and 17 had genetically associated tumors ( 5 MEN2A or MEN2B, 6 hypersecreting paraganglioma, 3 VHL disease, and 3 neurofibromatosis). Six pheochromocytomas were extra-adrenal, 11 were located in the left adrenal, and 15 in the right adrenal, while 14 were metastatic. Malignant disease was diagnosed on progressive metastatic disease at conventional imaging and/or positive metaiodobenzylguanidine (MIBG) in patients with previous diagnosis of pheochromocytoma. None of these patients had abnormal kidney function (creatininemia $<112 \mu \mathrm{mol} / \mathrm{l}$ or estimated glomerular filtration rate $(\mathrm{EGFR})>60 \mathrm{ml} / \mathrm{min}$ per $1.73 \mathrm{~m}^{2}$, MDRD formula). The study procedures were approved by the ethics committees of the centers recruiting patients, and all patients provided informed consent. Confirmation of pheochromocytoma was obtained by histological examination of surgically resected tumors.

\section{Laboratory analyses}

All blood samples were collected using a forearm venous cannula, with the patient kept supine for at least $20 \mathrm{~min}$ before sampling. Patients were instructed to fast and to abstain from caffeinated beverages or acetaminophen overnight. All samples were collected onto ice and centrifuged within $30 \mathrm{~min}$ after puncture. Plasma was kept at $-80{ }^{\circ} \mathrm{C}$ until analysis. Twenty-fourhour urinary metanephrines were collected into opaque bottles containing hydrochloric acid to ensure analyte stability.

The plasma samples were analyzed by HPLC with coulometric detection for free (4) and total metanephrines after acid hydrolysis (9). Metanephrines in urine were analyzed by HPLC as total hydrolysis-fractionated normetanephrine and metanephrine (15). Plasma catecholamines (norepinephrine and epinephrine) were analyzed using HPLC with amperometric detection (9).

\section{Statistical analysis}

The analysis of biomarker values was based on logtransformed data due to their skewed distribution. Reference limits were calculated as the antilog of the average \pm 2 s.D.s of $\log$ values. Associations with clinical factors were explored by $t$-tests, ANOVA, and linear correlations after log transformation.

Comparisons of sensitivity and specificity values for the various markers were performed on all available patient results after expressing each value as a ratio over its upper reference limit, adding the ratios of norepinephrine- and epinephrine-derived metabolites and defining cut-off values of 1 or 2 for this sum. Confidence intervals (CI) around sensitivity and specificity estimates were based on the binomial distribution. Receiver operating characteristic (ROC) curves were used to assess the performance of biochemical tests independently of cut-off choices.

Finally, the results of biochemical markers were integrated, after the transformation described above, into a logistic regression analysis to model diagnostic probability as a function of the biomarker measurement. The intercept of this logistic model $\left(\beta_{0 \text { sample }}\right)$ was corrected for the actual prevalence of pheochromocytoma in our population sample $\left(\mathrm{pr}_{\text {sample }}\right)$, thus recalibrating this estimate $\left(\beta_{0}\right.$ equi $)$ to a population having equiprobable risk of pheochromocytoma or not (uninformative 1:1 prior odds) (16). This was done by subtracting the logarithm of the actual prevalence odds: $\beta_{0}$ equi $=\beta_{0}$ sample - LOG $\left(\mathrm{pr}_{\text {sample }} /\left(1-\mathrm{pr}_{\text {sample }}\right)\right)$. Thereafter, we exploited the continuous aspect of biomarker values (expressed as sum of normalized ratios) to calculate a point estimate of the likelihood ratio for pheochromocytoma in a patient with a given test value $X$. This likelihood ratio was obtained as $\operatorname{LR}=\operatorname{EXP}\left(\beta_{0}\right.$ equi $\left.+\beta_{1 \text { sample }} \cdot X\right)$. This can be used to compute a post-test probability given the value of the biomarker in a target population with a priori prevalence of pheochromocytoma $p_{\text {pre }}$ by the usual formula: odds $\left(p_{\text {post }}\right)=\mathrm{LR} \cdot$ odds $\left(p_{\text {pre }}\right)$. This approach is appealing to incorporate in result interpretation the important disparity between pre-test probabilities in various patient subgroups (3). It assumes that the secretory pattern of pheochromocytoma does not differ across patient subgroups, and that the logarithm of the odds for pheochromocytoma increases continuously with the biomarker level. Statistical analyses were performed using STATA (v10, StataCorp, College Station, TX, USA).

\section{Results}

\section{Study population}

The 63 healthy subjects who provided samples for the study were aged 20-59 years (mean \pm s.D.: $32 \pm 10$ ), and $27 \%$ were females. The 181 symptomatic or predisposed controls in whom a pheochromocytoma was excluded were aged 7-86 (53 \pm 17$)$, and 56\% were females; $13 \%$ of them were known to have either a small adrenal incidentaloma or a hereditary predisposition to the tumor. The patients with evidence of pheochromo-

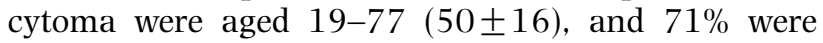
females and $85 \%$ had hypertension. None presented renal dysfunction $\left(\mathrm{EGFR}_{\mathrm{MDRD} \text {-formula }} 101 \pm 39 \mathrm{ml} / \mathrm{min}\right.$ per $1.73 \mathrm{~m}^{2}$ versus $94 \pm 46 \mathrm{ml} / \mathrm{min}$ per $1.73 \mathrm{~m}^{2}$ in 
controls, $P=0.40$ ). Finally, we received 404 samples referred from regional laboratories without detailed information on sampling procedure and/or clinical status, which were therefore not included in this study.

\section{Biochemical results}

The values observed for free and total plasma and urinary metanephrines and plasma catecholamines are shown in Fig. 2A-D. Their distribution was consistent with a log normal, allowing normalization by logarithmic transformation. These resulting reference limits are given in Table 1, along with the observed 97.5 percentiles characterizing healthy subjects and patient controls. There were no differences between the three groups of patient controls (hypertension with symptomatic paroxysms, genetic predisposition or follow-up after tumor resection and silent incidentaloma), except for a slightly higher concentration of plasma free metanephrine in the genetic predisposition patients (0.54 vs $0.43 \mathrm{nmol} / \mathrm{l}, P=0.04$, NS with Bonferroni's correction for test repetition). Thus, the data from the
181 patients were pooled in a single group. Therefore, the upper reference limits used in this study have been based on these 181 patients. Conversely, all norepinephrine-related analytes were lower in healthy subjects compared with the 181 control patients (relative differences $24-43 \%, P=0.02$ to $<0.001$ ), while analytes from the epinephrine lineage were comparable. This confirmed our decision not to include the healthy volunteers among the controls. The samples from the 404 patients not included in the study had slightly lower values than the samples from control patients for most markers, with a difference reaching statistical significance only for free metanephrine $(0.12$ vs $0.16 \mathrm{nmol} / \mathrm{l}, P=0.007)$. Among the 46 pheochromocytoma patients, there was no significant difference in any biological marker between the 33 cases referred retrospectively for established pheochromocytoma and the 13 cases identified prospectively on the basis of laboratory screening ( $t$-test on log-transformed values).

However, significant correlations of marginal importance, were found in control patients between age and plasma norepinephrine and total normetanephrine, and
A

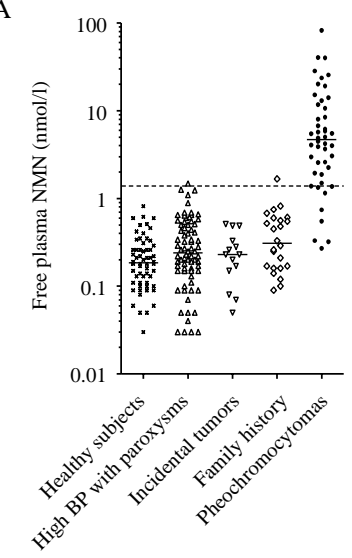

$\mathrm{C}$

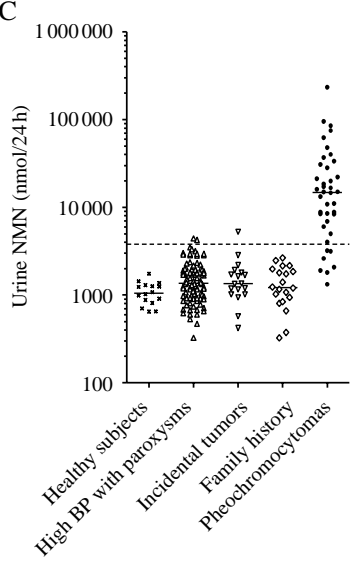

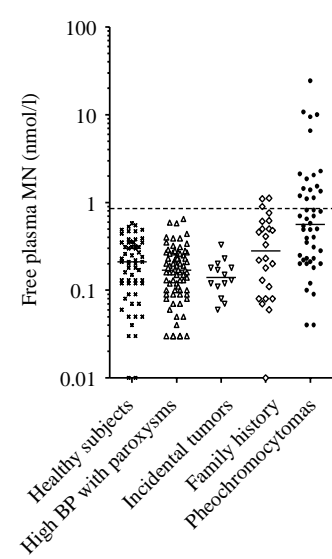

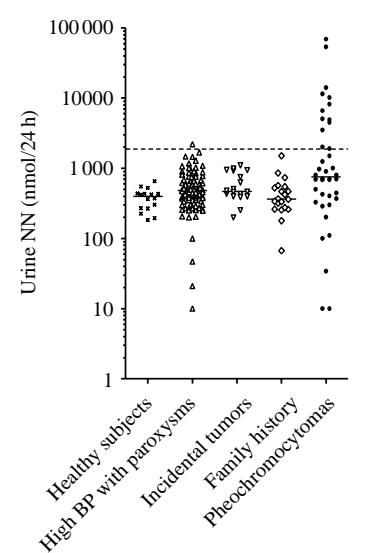

B
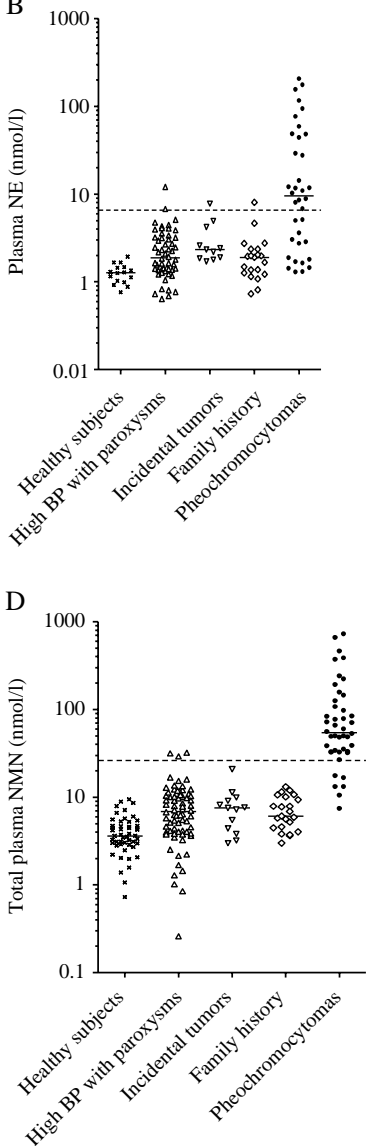
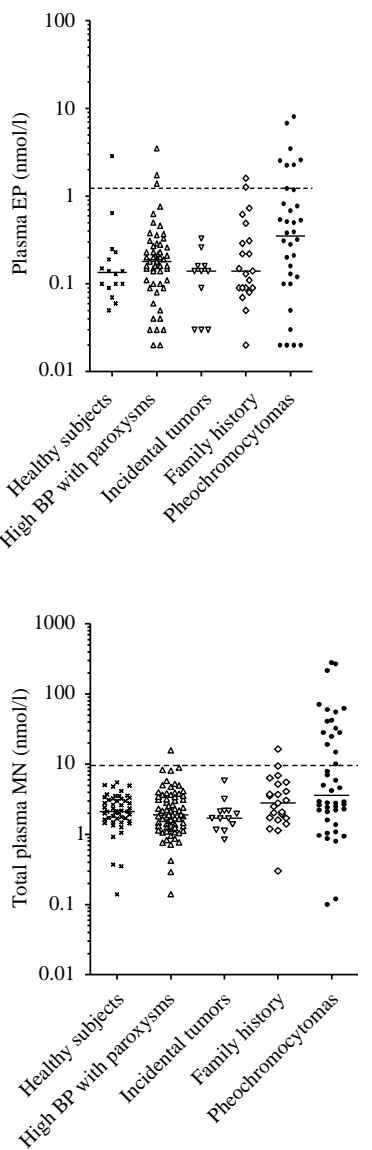

Figure 2 Distribution of biochemical markers for pheochromocytoma detection in the five study groups (63 healthy subjects, 133 symptomatic patients in whom pheochromocytoma was excluded, 23 patients with incidentaloma, 25 patients at familial risk, and 46 patients with established pheochromocytoma). (A) Plasma free metanephrines; (B) plasma catecholamines; (C) urinary metanephrines; (D) plasma total metanephrines. Dotted line, mean value; dash-dotted line, upper reference limit. 
Table 1 Percentiles of biochemical markers found in 63 healthy subjects and 181 hypertensive or predisposed patients (control patients).

\begin{tabular}{|c|c|c|c|c|c|c|}
\hline $\begin{array}{l}\text { Biochemical } \\
\text { test }\end{array}$ & Marker & Unit & $\begin{array}{l}\text { Healthy } \\
\text { subjects } \\
(P \text { 97.5) }\end{array}$ & $\begin{array}{l}\text { Control } \\
\text { patients } \\
(P 97.5)\end{array}$ & $\begin{array}{l}\text { Highest } \\
\text { control } \\
\text { patients }\end{array}$ & $\begin{array}{l}\text { Upper } \\
\text { reference } \\
\text { limit }\end{array}$ \\
\hline \multirow[t]{2}{*}{ Plasma free metanephrines } & Free normetanephrine & $\mathrm{nmol} / \mathrm{l}$ & 0.71 & 1.29 & 1.67 & 1.39 \\
\hline & Free metanephrine & $\mathrm{nmol} / \mathrm{l}$ & 0.56 & 0.92 & 1.12 & 0.85 \\
\hline \multirow[t]{2}{*}{ Plasma catecholamines } & Norepinephrine & $\mathrm{nmol} / \mathrm{l}$ & 1.94 & 7.99 & 12.11 & 6.55 \\
\hline & Epinephrine & $\mathrm{nmol} / \mathrm{l}$ & 2.85 & 1.72 & 3.52 & 1.23 \\
\hline \multirow[t]{2}{*}{ Urinary metanephrines } & Urinary normetanephrine & $\mathrm{nmol} / 24 \mathrm{~h}$ & 1759 & 3859 & 5249 & 3804 \\
\hline & Urinary metanephrine & $\mathrm{nmol} / 24 \mathrm{~h}$ & 653 & 1490 & 2200 & 1882 \\
\hline \multirow[t]{2}{*}{ Plasma total metanephrines } & Total normetanephrine & $\mathrm{nmol} / \mathrm{l}$ & 9.31 & 29.92 & 32.10 & 26.18 \\
\hline & Total metanephrine & $\mathrm{nmol} / \mathrm{l}$ & 5.35 & 11.26 & 16.40 & 9.61 \\
\hline
\end{tabular}

Upper reference limits of biochemical markers for pheochromocytoma detection are calculated as the mean plus two s.D.s of log-transformed values in hypertensive control patients.

urinary normetanephrine $\left(r^{2}=0.19,0.10\right.$, and 0.03 respectively). An association was found with sex, with female controls having urinary normetanephrine and metanephrine excretions respectively 24 and 38\% higher than males, explaining only 4 and $3 \%$ of overall variability. Therefore, we did not define genderspecific reference ranges. Plasma total metanephrine concentrations did not correlate with renal function $\left(r^{2}<0.01\right)$.

A cross-correlation analysis between biomarkers in pheochromocytoma patients showed expectedly positive intercorrelations between norepinephrine and its metabolites $\left(r^{2}=0.31-0.64\right)$ and between epinephrine and its metabolites $\left(r^{2}=0.04-0.56\right)$, and negative correlations between both metabolic lineages $\left(r^{2}\right.$ up to 0.38), meaning that the more pheochromocytomas release norepinephrine metabolites, the less they release epinephrine metabolites, and vice versa. No such intercorrelations were observed in controls $\left(r^{2}<0.14\right)$.

For two-thirds of the markers, our reference ranges differed significantly from those reported in the literature $(17,18)$, with plasma catecholamines and free metanephrines providing higher 97.5 percentile values in our controls $(P<0.05)$. Interestingly, our healthy subjects had lower plasma norepinephrine and total metanephrines, plasma free and urinary metanephrine than the controls (average differences
$24-43 \%, P<0.05)$. This underscores the importance of establishing local reference values for such biochemical parameters until inter-laboratory standardization is achieved (19).

\section{Diagnostic performance}

Pheochromocytoma tumors may overproduce norepinephrine or epinephrine either alone or simultaneously. Therefore, we expressed each individual measurement result as a fraction or a multiple of the corresponding upper reference value and summed up the pair of ratios obtained from related norepinephrineand epinephrine-derived markers. These 'sums of normalized ratios' are defined in Table 2. A cut-off value of 1 was chosen for the sum of normalized ratios to define a positive screening result favoring sensitivity to detect pheochromocytoma. With this 'detection cut-off', the sensitivities of plasma free and total metanephrines and urinary fractionated metanephrines were similar at $95-96 \%$ along with similar specificities of $86-91 \%$, while plasma catecholamines had a lower sensitivity at $72 \%$ for a specificity of $90 \%$ (Table 3 ).

In the case of positive results in one pair of markers, a more stringent interpretation favors the test specificity. A 'confirmation cut-off' was defined for a sum of normalized ratios exceeding 2 , raising the specificity of

Table 2 Diagnostic performances and likelihood ratio of biochemical markers for the detection of pheochromocytoma. Definition of the transformation of raw norepinephrine- and epinephrine-derived metabolite measurement results in a sum of normalized ratios over the respective upper reference limit.

\begin{tabular}{lll}
\hline Biochemical test & $\begin{array}{l}\text { Calculation for the sum of ratios } \\
\text { over reference limit }\end{array}$ & $\begin{array}{l}\text { Likelihood ratio for } \\
\text { pheochromocytoma }\end{array}$ \\
\hline Plasma free metanephrines & Normetanephrine/1.39+metanephrine/0.85 & EXP $(-4.1+3.5 \cdot X)$ \\
Plasma catecholamines & Norepinephrine/6.55+epinephrine/1.23 & EXP $(-1.4+1.6 \cdot X)$ \\
Urinary metanephrines & Normetanephrine/3804+metanephrine/1882 & EXP $(-5.2+3.7 \cdot X)$ \\
Plasma total metanephrines & Normetanephrine/26.18+metanephrine/9.61 & EXP $(-3.9+3.0 \cdot X)$ \\
\hline
\end{tabular}

The value of this index $(X)$ can then be transformed into a point likelihood ratio for the presence of pheochromocytoma. For combined tests, sums of normalized ratios for plasma free $(X)$ and urinary $(Y)$ metanephrines can be transformed into a positive likelihood ratio of $L R=E X P(-5.6+2.4 \cdot X+2.4 \cdot Y)$; for plasma total $(X)$ and urinary metanephrines: $\operatorname{LR}=\operatorname{EXP}(-6.4+2.4 \cdot X+2.7 \cdot Y)$; and for plasma free $(X)$ and total $(Y)$ metanephrines: $\operatorname{LR}=\operatorname{EXP}\left(-5.5+2.5 \cdot X_{\text {free }}+1.9 \cdot Y\right)$. 
Table 3 Comparison of diagnostic efficacy of biochemical markers for the detection of pheochromocytoma. Comparisons of sensitivity and specificity values for the four markers after expressing each value as a ratio over the upper reference limit by adding the ratios of norepinephrine- and epinephrine-derived metabolites at cut-off values of 1 or 2 for this sum.

\begin{tabular}{llrrr}
\hline Biochemical test & $\begin{array}{l}\text { Sensitivity }{ }^{\mathrm{a}} \text { with a } \\
\text { cut-off ratio at 1 }\end{array}$ & $\begin{array}{l}\text { Specificity }{ }^{\mathrm{a}} \text { with a } \\
\text { cut-off ratio at 1 }\end{array}$ & $\begin{array}{l}\text { Sensitivity }{ }^{\mathrm{a}} \text { with a } \\
\text { cut-off ratio at 2 }\end{array}$ & $\begin{array}{l}\text { Specificity }{ }^{\mathrm{a}} \text { with a } \\
\text { cut-off ratio at 2 }\end{array}$ \\
\hline Plasma free metanephrines & $44 / 46(96 ; 86-99)$ & $102 / 114(89 ; 83-94)$ & $35 / 46(76 ; 62-86)$ & $113 / 114(99 ; 95-100)$ \\
Plasma catecholamines & $26 / 36(72 ; 56-85)$ & $77 / 86(90 ; 81-94)$ & $20 / 36(56 ; 38-71)$ & $85 / 86(99 ; 94-100)$ \\
Urinary metanephrines & $38 / 40(95 ; 84-99)$ & $121 / 140(86 ; 80-91)$ & $34 / 40(85 ; 71-93)$ & $139 / 140(99 ; 96-100)$ \\
Plasma total metanephrines & $42 / 44(95 ; 85-99)$ & $98 / 108(91 ; 84-95)$ & $37 / 44(84 ; 71-92)$ & $104 / 107(97 ; 92-99)$ \\
\hline
\end{tabular}

${ }^{a}$ Numbers of patients (percentage; $95 \% \mathrm{Cl}$ ).

all markers to $97-99 \%$ but decreasing the sensitivity of plasma free metanephrines to $76 \%$, total metanephrines to $84 \%$, urinary metanephrines to $85 \%$, and plasma catecholamines to only $56 \%$ (Table 3 ). All the various combinations of tests associated two by two with a cut-off of 1 for the first test and 2 for the second test displayed specificity over $98 \%$, while sensitivity varied between 77 and $87 \%$.

Finally, the highest concentration observed for each test in controls without pheochromocytoma was taken as providing no false-positive results $(100 \%$ specificity, Table 1) giving a sensitivity of $69.6 \%$ to plasma free metanephrines, $68.2 \%$ to total metanephrines, $85.0 \%$ to urinary metanephrines, and only $36.6 \%$ to plasma catecholamines.

Among patients with false-negative results, a 13-year-old MEN2A was missed (metanephrine $0.82 \mathrm{nmol} / \mathrm{l}$ ) with a tumor size of $<2 \mathrm{~cm}$; a hypertensive patient operated for pheochromocytoma in the left adrenal relapsed on the right adrenal and had only elevated plasma free normetanephrine without alterations of total and urinary metanephrines. We also found an isolated increase of plasma total normetanephrine in a patient with VHL disease and metastatic pheochromocytoma. A non-hypertensive MEN2B patient had only plasma total metanephrine elevated with an adrenal tumor size of $3 \mathrm{~cm}$.

The areas under the ROC curve were 0.9925 (95\% CI 0.978-1.000) for plasma free metanephrines, 0.989 (0.971-1.000) for total metanephrines, 0.9941 (0.986-1.000) for urinary metanephrines, and 0.858 (0.760-0.956) for plasma catecholamines. Pairwise comparisons showed that urinary metanephrines and plasma total and free metanephrines produced similar areas under the ROC curve (Fig. 3).

\section{Likelihood ratios}

Finally, we attempted to express the likelihood ratio as a function of actual values of pairs of related biomarkers for computing post-test probabilities in a target population having given pre-test probability (Table 2). Such models explain about $75 \%$ of the variability in diagnostic outcomes (based on McFadden's pseudo- $R^{2}$ ) except for catecholamines (40\%). A graphical representation (Fig. 4) confirms that the models adequately smooth the stepping sequence of piecewise predictive values except in the case of plasma catecholamines. For this marker, the overlap between cases and controls casts doubts about the negative predictive value of even very low results, and/or the assumption underlying that the logistic model (linearity of the relationship between biomarker and log odds of the risk of pheochromocytoma) may not be confirmed by the data.

In a patient with a given prior probability of pheochromocytoma, post-test probability odds can be deduced from biomarker values by multiplying prior odds by the LR. For example, a patient with newly diagnosed hypertension can be considered only at $0.2 \%$ risk of pheochromocytoma (20); measuring total normetanephrine at $42 \mathrm{nmol} / \mathrm{l}$ and total metanephrine at $5 \mathrm{nmol} / \mathrm{l}$ gives a sum of normalized ratios of 2.13 $(=42 / 26.18+5 / 9.61)$, hence a likelihood ratio of 11.2 $(=\operatorname{EXP} \quad(-3.862+2.954 \cdot 2.13))$, which would transform his/her prior odds from 0.002 into 0.022 , and hence a posterior probability of $2 \%$. The same laboratory results in a patient investigated for

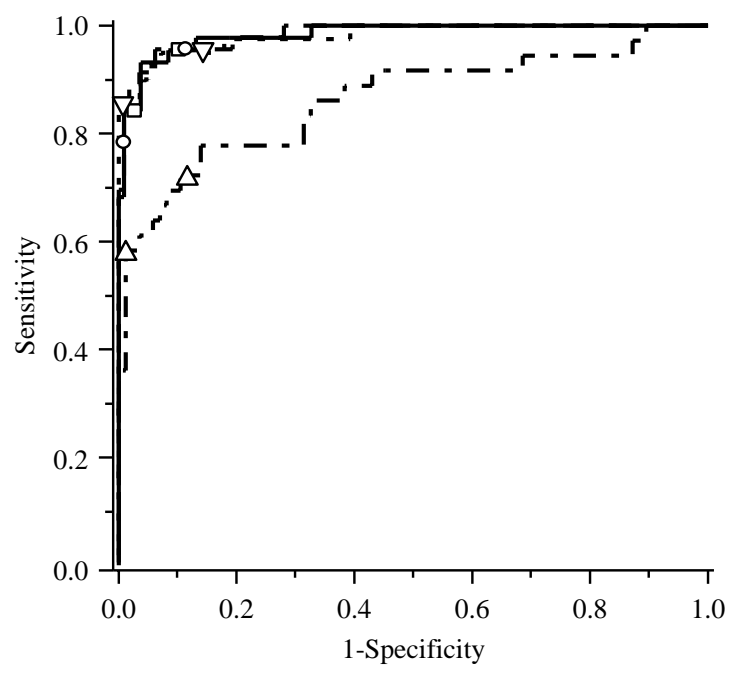

Figure $3 \mathrm{ROC}$ curves for the diagnostic performance of combined plasma free metanephrines (dashed line), urinary metanephrines (dotted line), plasma total metanephrines (continuous line), and plasma catecholamines (dash-dotted line) determinations. The symbols indicate cut-off values of 1 and 2 for the sum of norepinephrine- and epinephrine-derived metabolites, expressed as a fraction of the upper reference range (see text). 

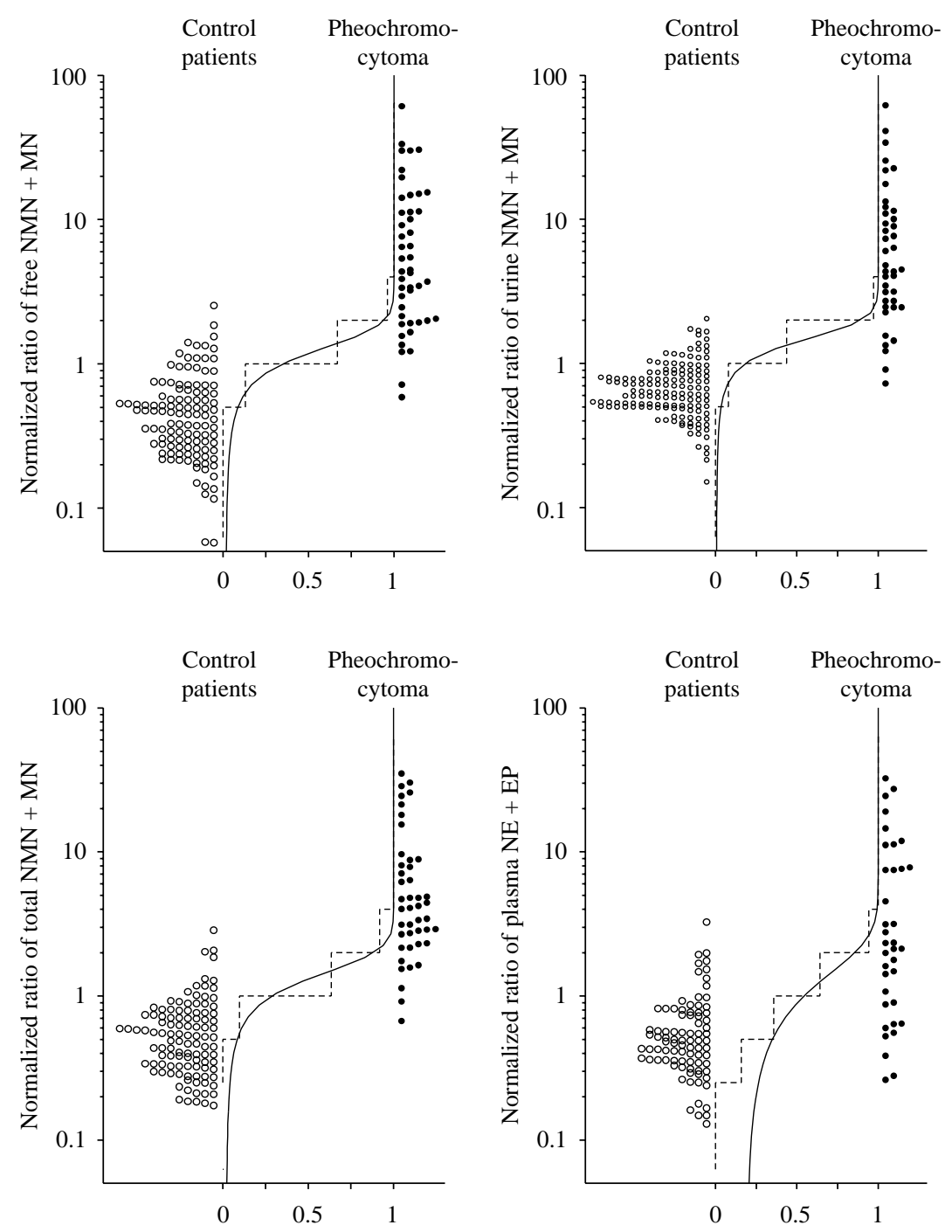

\begin{abstract}
Figure 4 Distribution of individual sums of norepinephrine- and epinephrine-derived metabolites, expressed as a fraction of the upper reference range, in controls (open symbols) and pheochromocytoma patients (closed symbols). Stepwise dashed lines indicate (along the $X$-axis) the fraction of pheochromocytoma cases observed per slice interval of the score $(<0.5,0.5-1,1-2,2-4$, and $>4$ ), adjusted for the respective total number of controls and cases. Continuous curves depict the calculated probability of pheochromocytoma at a given score value ( $Y$-axis) according to a logistic regression model (see text).
\end{abstract}

incidentaloma would transform his/her prior probability of $5 \%$ (21) into a posterior probability of $37 \%$. In an adult freshly diagnosed for MEN2B, they would raise his/her prior probability from already as high as $50 \%$ (22) up to $92 \%$. Results of two pairs of determinations performed in a patient may even be combined in a logistic model adjusting for the cross-correlation between analytes (see footnote Table 2). These combinations increase the percentage of variability explained (i.e. the diagnostic prediction) from 75 to $83-88 \%$ consistently with moderate intercorrelation between biomarkers.

\title{
Discussion
}

Our results indicate similarly high sensitivity of plasma total or free or urinary metanephrines for the diagnosis of pheochromocytoma. All three measurements provide clearly better clues to detect this tumor compared with

the classical determination of plasma catecholamines. Their areas under ROC curves are similar when taking as controls patients with treatment-resistant hypertension and paroxysmal symptoms, silent incidentalomas, familial syndromes without tumor at the time of testing, or hereditary predisposition with remission after tumor resection. These control groups had similar concentrations of all biomarkers investigated. To date, none of these tests appear able to detect pheochromocytoma before the others at an earlier time of tumor development.

In our algorithm, we have proposed to screen for pheochromocytoma with a single biochemical test (among the three tests based on metanephrines), taking as the cut-off point a sum of normalized ratios over upper reference limits of 1 , corresponding to a sensitivity of at least $95 \%$. A score below 1 can be considered as negative with fair confidence. However, since $20-25 \%$ of initial test results will exhibit a score over 1 , a confirmatory test based on a different biomarker is suitable. Choosing a 
cut-off of 2 for these confirmatory scores raises the specificity to at least $95 \%$. A score below 2 indicates the absence of the disease, while a value over 2 would predict pheochromocytoma.

Pheochromocytomas affect not more than $0.1-0.4 \%$ of the hypertensive population (20). Physicians often refer patients with treatment-resistant hypertension to the laboratory for analyzing catecholamines and/or their metabolites. Bravo reported that biochemical testing is mainly used to exclude the diagnosis of pheochromocytoma (23); the fear of missing a pheochromocytoma, easily cured by surgery, calls for $100 \%$ sensitivity. Biochemical screening does not always follow a rational strategy, even in hospitals where physicians are more prone to follow policies. Indeed, we still receive a number of inadequate requests for catecholamine measurement during a hypertensive crisis to 'exclude pheochromocytoma'. Consequently, the laboratory must propose not only the most sensitive test to ensure not to miss the disease, but also a conveniently specific test for disease confirmation in case of positive screening. However, recommendations remain controversial. Sawka estimates that the measurement of 24-h catecholamine and metanephrines has appropriate sensitivity and specificity to detect sporadic pheochromocytomas (6). Conversely, Eisenhofer \& Raber propose plasma free metanephrines as the first choice biomarker for tumor screening (3-5, 24). A universal guideline for the biochemical investigation of pheochromocytoma has been proposed at the first pheochromocytoma symposium, and it relies on the determination of urinary metanephrines or plasma free metanephrines (3) as the first choice for screening.

Plasma free metanephrines are difficult to measure with accuracy because their concentrations are 2000fold lower than those of urinary metanephrines. Some US centers appear to concentrate their expertise on measuring free metanephrines among other tests for pheochromocytoma diagnosis. In Switzerland and France, a large number of laboratories only measure catecholamines in plasma and urine and/or metanephrine in urine. In addition, variable reference intervals are used across laboratories (when not simply taken from the literature), and 24-h urine collection is poorly accurate, especially in children, which further limits its usefulness in outpatients. Overnight urine collection or correction by creatininemia has been proposed, but it does not always correct urine collection uncertainty (25). However, urinary metanephrine and catecholamine samples are stable if acidified below $\mathrm{pH}$ 3.5, and they can be readily posted.

An advantage of blood collection over urine collection is the absence of volume and time recording. Total metanephrines are relatively easy to measure and rather stable (we found no loss of blood-conjugated metanephrines during repetitive measurement of a sample stored at $4{ }^{\circ} \mathrm{C}$ for several days) compared with the limited stability of plasma free metanephrines or whole blood catecholamines (26). Moreover, total metanephrines fluctuate minimally over the day, either in healthy volunteers or in pheochromocytoma patients $(9,27)$, and are almost insensitive to stress compared with catecholamines (directly released from sympathetic nerves) or with free metanephrines (reactive to a lesser extent). Interestingly, pheochromocytoma cell cultures release free metanephrines (28), however without sulfoconjugating them, since concentrations of intracellular or secreted free metanephrines are equal to those of total metanephrines (data not shown). Sulfoconjugation of metanephrines secreted by the tumor is thus a slow process ensuring a good stability of plasma total concentrations. False-positive results associated to medications are less frequently encountered with free metanephrines than with other biomarkers (29). Peyrin et al. found that among the usual antihypertensive drugs, only angiotensin-converting enzyme inhibitors and diuretics induce infrequently moderate increases in total normetanephrine, however with minor consequences in result interpretation (17). All these reasons explain that total plasma metanephrines appear as specific as other biomarkers measured for pheochromocytoma detection. Nevertheless, as metabolic end products that are principally eliminated through the kidneys, they may increase during renal dysfunction (creatininemia $>170 \mu \mathrm{mol} / \mathrm{l}$ or creatinine clearance $<30 \mathrm{ml} / \mathrm{min}(10-12)$ ). Therefore, the knowledge of the patient's renal status is requested to interpret plasma total metanephrines. This study did not include patients with renal impairment. In the case of renal failure, the screening algorithm proposed by Sawka (adjustment of the cut-off ratio for a positive result of plasma free metanephrines and combination with 24-h urinary metanephrines) seems to be most appropriate and cost-effective for detecting pheochromocytoma (30). The sulfotransferase SULT1A3 is very active in the gastrointestinal tract, and may produce conjugated metanephrines after consumption of foods (31). Dietary influences have been reported not to influence plasma total metanephrines (32), whereas a recent publication has established that a standardized meal rich in metanephrines (including six bananas) may induce an increase of plasma-deconjugated normetanephrine, plasma-deconjugated and free methoxytyramine, and urine-deconjugated normetanephrine (33).

Among other limitations, this study was retrospective, unblinded, and included only 46 patients with histopathologically confirmed pheochromocytoma. Furthermore, measurement values were not available for all parameters in all patients (urinary catecholamines, available for a minority of pheochromocytoma patients, were not included in the study). Follow-up of 3 years or more may not be sufficient to definitely exclude pheochromocytoma in control patients. Finally, the assessment of sensitivity, specificity, and likelihood ratio for the diagnosis of pheochromocytoma was not validated in independent patients, but 
in the same patients was used to establish reference ranges, and so it is likely that the test performance is overestimated. On the other hand, this study had the merit of including a mixture of samples originating from patients with various signs reminiscent of pheochromocytoma, a representative case-mix usually seen in specialized laboratories (14). The logistic regression approach that we have proposed to calculate a point estimate of the patient's positive likelihood ratio, based on his/her test results, probably captures the maximum information from this case-control study; however, it should certainly not be taken with undue overprecision, and should still be considered experimental, deserving prospective validation. While the reasonable assumptions underlying this approach are contradicted by no known facts, they are not confirmed either. Our study indicates that plasma free and total metanephrines and urinary metanephrines are equivalent for assessing a pheochromocytoma. The combination of two of these tests probably represents the best approach to screen and then confirm biochemically the suspicion of a tumor. We propose that the combination of both blood tests in a single sample is certainly the most convenient alternative for the patient and a reliable method for the physician to assess/exclude a pheochromocytoma.

\section{Declaration of interest}

The authors declare that there is no conflict of interest that would prejudice the impartiality of this scientific work.

\section{Funding}

This study was funded by the French Society of Hypertension.

\section{References}

1 Manger WM \& Gifford RW. In Clinical and Experimental Pheochromocytoma, edn 2. Cambridge: Blackwell Science, 1996.

2 Plouin PF, Duclos JM, Menard J, Comoy E, Bohuon C \& Alexandre JM. Biochemical tests for the diagnosis of pheochromocytoma: urinary versus plasma determinations. BMJ 1981 282 853-854.

3 Pacak K, Eisenhofer G, Ahlman H, Bornstein SR, GimenezRoqueplo AP, Grossman AB, Kimura N, Mannelli M, McNicol AM \& Tischler AS. International symposium on pheochromocytoma. Pheochromocytoma: recommendations for clinical practice from the First International Symposium October 2005. Nature Clinical Practice. Endocrinology E Metabolism 20073 92-102.

4 Lenders JW, Pacak K, Walther MM, Linehan WM, Mannelli M, Friberg P, Keiser HR, Goldstein DS \& Eisenhofer G. Biochemical diagnosis of pheochromocytoma: which test is best? Journal of the American Medical Association 2002287 1427-1434.

5 Raber W, Raffesberg W, Bischof M, Scheuba C, Niederle B, Gasic S, Waldhäusl W \& Roden M. Diagnostic efficacy of unconjugated plasma metanephrines for the detection of pheochromocytoma. Archives of Internal Medicine $20001602957-2963$.

6 Sawka AM, Jaeschke R, Singh RJ \& Young WF. A comparison of biochemical tests for pheochromocytoma: measurement of fractionated plasma metanephrines compared to the combination of 24-hour urinary metanephrines and catecholamines. Journal of Clinical Endocrinology and Metabolism 200388 553-558.

7 Lenders JW, Willemsen JJ, Eisenhofer G, Ross HA, Pacak K, Timmers HJ \& Sweep CG. Is supine rest necessary before blood sampling for plasma metanephrines? Clinical Chemistry $2007 \mathbf{5 3}$ 352-354.

8 Boyle JG, Davidson DF, Perry CG \& Connell JM. Comparison of diagnostic accuracy of urinary free metanephrines, vanillyl mandelic acid, and catecholamines and plasma catecholamines for diagnosis of pheochromocytoma. Journal of Clinical Endocrinology and Metabolism $2007924602-4608$.

9 Grouzmann E, Fathi M, Gillet M, de Torrenté A, Cavadas C, Brunner H \& Buclin T. Disappearance rate of catecholamines, total metanephrines, and neuropeptide $\mathrm{Y}$ from the plasma of patients after resection of a pheochromocytoma. Clinical Chemistry 2001 47 1075-1082.

10 Eisenhofer G, Huysmans F, Pacak K, Walther MM, Sweep FC \& Lenders JW. Plasma metanephrines in renal failure. Kidney International $200567668-677$.

11 Mornex R, Peyrin L, Pagliari R \& Cottet-Emard JM. Measurement of plasma methoxyamines for the diagnosis of pheochromocytoma. Hormone Research 199136 220-226.

12 Marini M, Fathi M \& Vallotton M. Determination of serum metanephrines in the diagnosis of pheochromocytoma. Annales d'Endocrinologie 199454 337-342.

13 Lenders JW, Keiser HR, Goldstein DS, Willemsen JJ, Friberg P, Jacobs MC, Kloppenborg PW, Thien T \& Eisenhofer G. Plasma metanephrines in the diagnosis of pheochromocytoma. Annals of Internal Medicine 1995123 101-109.

14 Knottnerus JA \& Buntinx F. In The Evidence Base of Clinical Diagnosis, Eds JA Knottnerus \& F Buntinx, Chichester: John Wiley \& Sons, 2008.

15 Kairisto V, Koskinen P, Mattila K, Puikkonen J, Virtanen A, Kantola I \& Irjala K. Reference intervals for 24-h urinary normetanephrine, metanephrine, and 3-methoxy-4-hydroxymandelic acid in hypertensive patients. Clinical Chemistry 199238 416-420.

16 Morise AP, Diamond GA, Detrano R, Bobbio M \& Gunel E. The effect of disease-prevalence adjustments on the accuracy of a logistic prediction model. Medical Decision Making 199616 133-142.

17 Peyrin L, Cottet-Emard JM, Pagliari R, Cottet-Emard RM, Badet C \& Mornex R. Plasma methoxyamines assay: a practical advance for the diagnosis of pheochromocytoma. Pathologie et Biologie 1994 42 847-854.

18 Peyrin L \& Mornex R. Biological diagnosis of pheochromocytoma: impact of technological improvement. Annales de Biologie Clinique $199351835-865$.

19 Pillai D, Ross HA, Kratzsch J, Pedrosa W, Kema I, Hoad K, Rouaix N, Fathi M, Nader H, Mathian B \& Grouzmann E. Proficiency test of plasma free and total metanephrines: report from a study group. Clinical Chemistry and Laboratory Medicine 200947 786-790.

20 Lo CY, Lam KY, Wat MS \& Lam KS. Adrenal pheochromocytoma remains a frequently overlooked diagnosis. American Journal of Surgery $2000179212-215$.

21 Mantero F, Terzolo M, Arnaldi G, Osella G, Masini AM, Alì A, Giovagnetti M, Opocher G \& Angeli A. A survey on adrenal incidentaloma in Italy. Study Group on Adrenal Tumors of the Italian Society of Endocrinology. Journal of Clinical Endocrinology and Metabolism 200085 637-644.

22 Hansford JR \& Mulligan LM. Multiple endocrine neoplasia type 2 and RET: from neoplasia to neurogenesis. Journal of Medical Genetics 200037 817-827.

23 Bravo EL. Diagnosis of pheochromocytoma. Reflections on a controversy. Hypertension $1991 \mathbf{1 7} 742-744$.

24 Goldstein DS, Eisenhofer G, Flynn JA, Wand G \& Pacak K. Diagnosis and localization of pheochromocytoma. Hypertension 200443 907-910. 
25 Héron E, Chatellier G, Billaud E, Foos E \& Plouin PF. The urinary metanephrine to creatinine ratio in the diagnosis of pheochromocytoma. Annals of Internal Medicine 1996125 300-303.

26 Willemsen JJ, Sweep CGJ, Lenders JWM \& Ross HA. Stability of plasma free metanephrines during collection and storage as assessed by an optimized HPLC method with electrochemical detection. Clinical Chemistry 200349 1951-1953.

27 Hoizey G, Lukas-Croisier C, Frances C, Grulet H, Delemer B, Millart H, Devillier P \& Caron J. Study of diurnal fluctuations of plasma methoxyamines in healthy volunteers. Clinical Endocrinology 200256 119-122.

28 Eisenhofer G, Keiser H, Friberg P, Mezey E, Huynh TT, Hiremagalur B, Ellingson T, Duddempudi S, Eijsbouts A \& Lenders JW. Plasma metanephrines are markers of pheochromocytoma produced by catechol-O-methyltransferase within tumours. Journal of Clinical Endocrinology and Metabolism 1998 $832175-2185$.

29 Eisenhofer G, Goldstein DS, Walther MM, Friberg P, Lenders JW, Keiser HR \& Pacak K. Biochemical diagnosis of pheochromocytoma: how to distinguish true-from false-positive test results. Journal of Clinical Endocrinology and Metabolism $2003 \mathbf{8 8}$ 2656-2666.
30 Sawka AM, Gafni A, Thabane L \& Young WF Jr. The economic implications of three biochemical screening algorithms for pheochromocytoma. Journal of Clinical Endocrinology and Metabolism 200489 2859-2866.

31 Goldstein DS, Mezey E, Yamamoto T, Aneman A, Friberg P \& Eisenhofer G. Is there a third peripheral catecholaminergic system? Endogenous dopamine as an autocrine/paracrine substance derived from plasma DOPA and inactivated by conjugation Hypertension Research 199518 S93-S99.

32 Pagliari R, Cottet-Emard JM \& Peyrin L. Determination of free and conjugated normetanephrine and metanephrine in human plasma by high-performance liquid chromatography with electrochemical detection. Journal of Chromatography 1991563 23-36.

33 de Jong WH, Eisenhofer G, Post WJ, Muskiet FA, de Vries EG \& Kema IP. Dietary influences on plasma and urinary metanephrines: implications for diagnosis of catecholamine-producing tumors. Journal of Clinical Endocrinology and Metabolism 200994 2841-2849.

Received 1 February 2010

Accepted 7 February 2010 